\title{
Capnography for monitoring non-intubated spontaneously breathing patients in an emergency room setting
}

\author{
C V Egleston, H Ben Aslam, M A Lambert
}

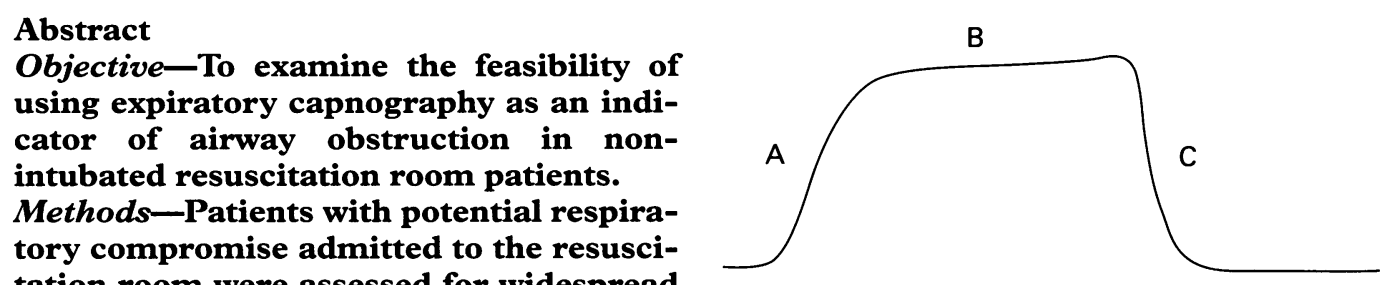
tory compromise admitted to the resuscitation room were assessed for widespread expiratory wheeze. This was taken as clinical evidence of airways obstruction. Expiratory capnograms of these patients and patients who had no wheeze were obtained. The traces were analysed for basic morphology and where appropriate the slope ratio (SR) between phase 1 (S1) and phase 2 (S2) of the trace was obtained. Results-Thirty eight patients with a variety of clinical conditions causing potential or actual respiratory impairment were studied. All patients tolerated the nasal capnogram cannulae. Twelve had no clinical evidence of airway obstruction and all had capnograms with normal morphology. Eleven of these were analysed further. The mean value for SR was 7.57 (SEM $0.18), 95 \%$ confidence interval 6.37 to 8.77 . Twenty six patients had clinical evidence of airway obstruction. The capnograms showed obstructive "shark's fin" morphology. Fourteen of these were analysed to determine SR. The mean value was 31.9 (4.46), $95 \%$ CI 22.9 to 40.8 . There was a significant difference in the mean value for $S R$ between the two groups $(P<0.001)$.

Conclusions-Capnography may be used as a means of continuous respiratory monitoring in non-intubated acutely ill patients. Capnogram analysis may be used to indicate airway obstruction in these patients. Further work is required to correlate curve indices to degree of airway obstruction.

(F Accid Emerg Med 1997;14:222-224)

\section{Accident an}

Department, Norfolk \& Norwich Hospital, Brunswick Road, Norwich

C V Egleston

H Ben Aslam

M A Lambert

Correspondence to: C V Egleston, c/o Accident and Emergency Department, Southampton General Hospital, Tremona Rd, Southampton SO16 6YD.

Accepted for publication 28 February 1997
Keywords: capnography; airway obstruction; nonintubated patients

Capnometry, the measurement of the concentration of atmospheric carbon dioxide, was first developed during the second world war as a means of monitoring the internal environment of submarines. ${ }^{1}$ Its use in medicine dates from the 1950 s when capnometers were first used experimentally to measure expired $\mathrm{CO}_{2}$ during anaesthesia. However, it was not until the early 1980 s, with the development of smaller practical machines, that capnography (the plotting of
Figure 1 A normal capnogram trace.

a trace obtained by continuous recording of expired $\mathrm{CO}_{2}$ ) entered routine anaesthetic practice. ${ }^{2}$ Today capnography is considered to be essential to monitor metabolic and respiratory function in anaesthetised patients. ${ }^{3}$ Its main use is to detect adverse airway events such as endotracheal tube displacement and blockage.

The normal capnogram has an almost rectangular shape (fig 1) with a rapid upstroke (A) corresponding to the increasing concentrations of $\mathrm{CO}_{2}$ in gas from more distal airways, an almost horizontal plateau (B) corresponding to exhalation of gas from alveoli with near homogeneous concentrations of $\mathrm{CO}_{2}$, and a downstroke (C) at the time of inhalation in the respiratory cycle. With airway obstruction it is recognised that the capnogram curve changes shape so that the initial upstroke is more gradual. In addition there is no proper plateau formation. ${ }^{134}$ These changes give rise to the so called "shark's fin" morphology capnogram in patients with airway obstruction (fig 2). Other workers have correlated certain curve indices such as the slopes of different parts of capnogram curve, as well as areas subtended by different parts of the curve, with different degrees of airway obstruction quantified by spirometric findings. ${ }^{4}$

We performed this study with the aim of determining the feasibility of using capnography to monitor non-intubated patients in a resuscitation room setting. We also wished to correlate clinical evidence of airway obstruction with capnogram curve indices.

\section{Methods}

Patients admitted for treatment to the resuscitation room with conditions which threatened or resulted in respiratory compromise were opportunistically recruited by the authors. These patients were assessed for the presence of expiratory wheeze which was taken to indicate lower airway obstruction. During treatment for their presenting condition patients had a capnogram nasal cannula (fig 3 ) inserted in one of their nostrils. This cannula consists of rigid walled flexible plastic tubing which is encased at the patient's end in a soft 


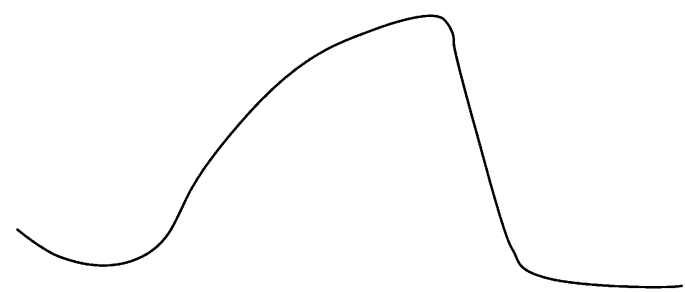

Figure 2 A capnogram showing obstructive "shark's fin" morphology.

plastic cylinder through which the patient can breathe. The cannula was kept in place by taping the tubing to the patient's upper lip. One cannula was used per patient. A 30 second capnography trace was obtained (Datex Normocap 200, Instrumentation Corp, Helsinki, Finland). The trace morphology was assessed in the wheezing and non-wheezing group to determine if it showed a normal or obstructive (that is, Shark's fin) pattern.

Suitable traces were submitted to further analysis to determine the slopes of the upstroke phase (S1) and plateau phase (S2) of the capnogram (fig 4). S1 was measured between 0 and 0.2 seconds of the capnogram, and S2 was measured between 0.8 and 1.2 seconds. The slope ratio (SR) was obtained by the formula $\mathrm{SR}=\mathrm{S} 2 / \mathrm{S} 1 \times 100$.

\section{STATISTICS}

Capnograms were not considered suitable for slope ratio analysis if individual traces lasted less than 0.8 seconds or more than 3 seconds, or if they were extremely deformed by artefact. In addition traces were excluded if the end tidal $\mathrm{CO}_{2}$ was less than $3.72 \mathrm{kPa}$. Previous work has shown that measurement of the slope ratio is unreliable in these circumstances. ${ }^{4}$ The mean

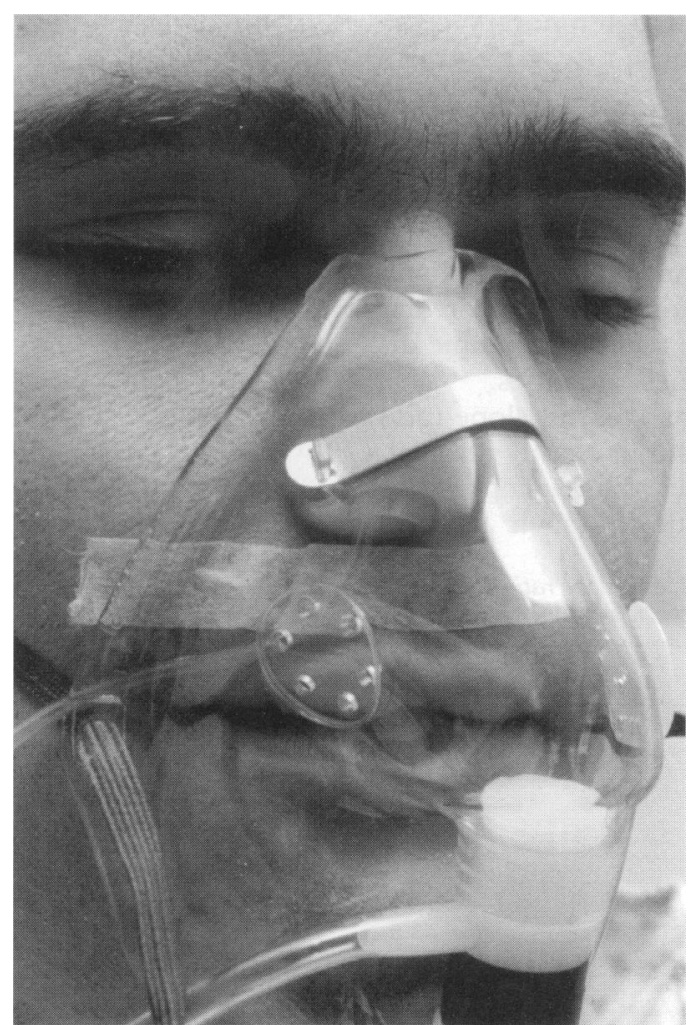

Figure 3 Nasal capnogram cannula and oxygen mask in place on model.

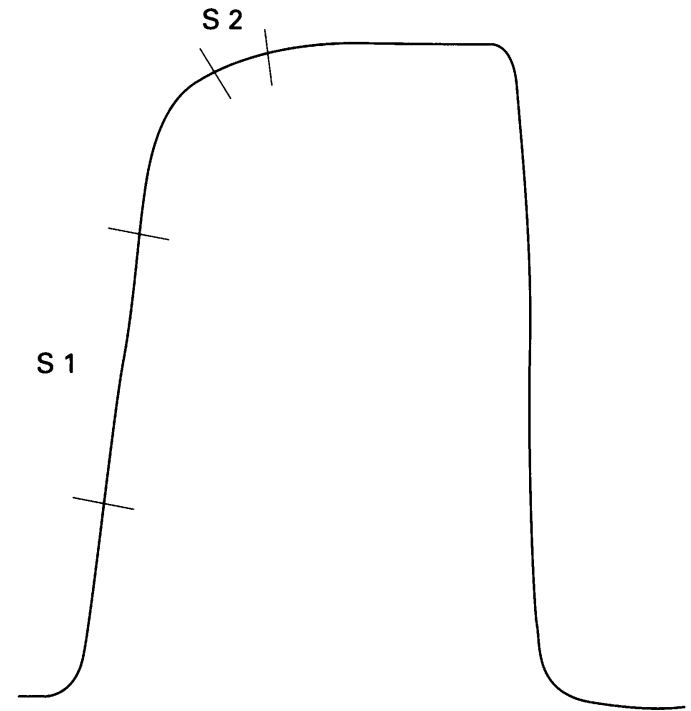

Figure 4 Measurement of $S 1$ and $S 2$ of the capnogram trace.

slope ratios of the normal and obstructed groups were obtained. The $t$ test for the comparison of two independent means was applied.

\section{Results}

Thirty eight patients presenting to the resuscitation room with conditions which could result in respiratory compromise were included in the study. Twenty six patients had expiratory wheeze. All of these had obstructive pattern capnograms (that is, slow climbing phase 1 of the capnogram and no proper plateau formation). The diagnoses of the wheezing group are shown in table 1.

Twelve patients had no expiratory wheeze; all of these had normal rectangular capnogram morphology. The diagnoses of the normal morphology group are shown in table 2 .

The median age of the normal morphology group was 32 (range 20 to 62) and in the obstructed group it was 37 (range 16 to 90 ). The male to female ratio was 8:4 in the normal group and 11:15 in the obstructed morphology group.

Using the criteria explained above, 11 traces of the normal morphology group and 14 of the obstructed morphology group were suitable for slope ratio determination. The mean slope ratio of the normal (non-wheezing) group was 7.57 (SEM 0.18), 95\% confidence interval (CI) 6.37 to 8.77 . In the obstructed morphology group the mean slope ratio was 31.9 (4.46), $95 \%$ CI 22.9 to 40.8 . S1, S2, and slope ratio values for the two groups are shown in tables 3 and 4 . The difference between the means for the normal and obstructed group slope ratio was statistically significant $(\mathrm{P}<<0.001)$.

\section{Discussion}

There are various options available for the assessment of airway obstruction in the acutely ill patient: clinical assessment, peak flow estimation (in the case of asthma), and spirometry. There are obvious drawbacks to these methods. In the case of clinical assessment, the emergency physician may rely on the presence of expiratory wheeze to indicate the presence of lower airway obstruction; however, as is well 
Table 1 Primary diagnoses of patients with expiratory wheeze

\begin{tabular}{ll}
\hline Primary diagnosis & Number of cases \\
\hline Asthma & 12 \\
Pulmonary oedema & 7 \\
COPD & 2 \\
Pneumonia & 2 \\
Smoke inhalation & 2 \\
Self poisoning & 1 \\
\hline
\end{tabular}

COPD, chronic obstructive pulmonary disease.

Table 2 Primary diagnoses of patients with no expiratory wheeze

\begin{tabular}{ll}
\hline Primary diagnosis & Number of cases \\
\hline Self poisoning & 6 \\
Smoke inhalation & 2 \\
Pneumothorax & 1 \\
Head injury & 1 \\
Facial burns & 1 \\
Angina & 1 \\
\hline
\end{tabular}

Table 3 The values for S1, S2, and slope ratio (SR) for the normal morphology (non-obstructed) group

\begin{tabular}{lll}
\hline$S 1$ & $S 2$ & $S R$ \\
\hline 4 & 0.5 & 12.5 \\
4.7 & 0.33 & 6.6 \\
4.7 & 0.33 & 7.02 \\
4 & 0.33 & 8.25 \\
6 & 0.33 & 5.5 \\
4 & 0.33 & 8.25 \\
4 & 0.2 & 5 \\
5 & 0.33 & 6.6 \\
3 & 0.25 & 8.33 \\
3.5 & 0.3 & 8.57 \\
3 & 0.2 & 6.7 \\
\hline
\end{tabular}

known that the intensity of wheeze does not necessarily correlate with the degree of airway obstruction and wheeze may be absent altogether in low air entry states. ${ }^{5}$ Peak flow estimation is very dependent on good patient technique and requires the temporary cessation of oxygen and nebulised drug treatment. Spirometry is too cumbersome and requires good patient technique and a considerable degree of observer expertise to be a useful form of patient assessment in the emergency situation. An ideal form of respiratory monitoring would not rely heavily on patient compliance or require the interruption of oxygen or drug treatment, while giving a real time graphic display. Capnography has these qualities.

We chose a curve index (that is, slope ratio) which could be measured easily from a capnography trace rather than using indices mentioned in other studies (such as areas

Table 4 The values for S1, S2, and slope ratio (SR) for the abnormal morphology (obstructed group)

\begin{tabular}{lll}
\hline$S 1$ & $S 2$ & $S R$ \\
\hline 2 & 0.5 & 25 \\
3 & 01.1 & 36.7 \\
3 & 0.50 & 16.7 \\
3 & 0.5 & 16.7 \\
2.5 & 0.5 & 16 \\
2 & 1.25 & 62.5 \\
2 & 1 & 50 \\
3 & 0.5 & 16.7 \\
2 & 1.25 & 62.5 \\
3 & 1 & 33 \\
3 & 0.67 & 22.3 \\
3 & 1.33 & 44.3 \\
3 & 0.67 & 22.3 \\
3 & 0.67 & 22.3 \\
\hline
\end{tabular}

under different sections of the capnography curve) which require computer analysis. ${ }^{4}{ }^{6} \mathrm{We}$ did this in order to show that any clinician with a capnograph which is capable of generating a hard copy may determine the slope ratio without recourse to more sophisticated equipment. In previous studies the slope ratio has been correlated with different values of forced expiratory volume in an experimental setting. ${ }^{4}$ We know of one other study where end tidal $\mathrm{CO}_{2}\left(\mathrm{PetCO}_{2}\right)$ was compared with arterial $\mathrm{CO}_{2}$ $\left(\mathrm{PaCO}_{2}\right)$ in emergency room patients. ${ }^{7}$ This study confirmed what was already known for a long time in anaesthetic practice ${ }^{3}$; that in patients with pulmonary disease $\mathrm{PetCO}_{2}$ does not correlate reliably with $\mathrm{PaCO}_{2}$ (the two values diverge, $\mathrm{PetCO}_{2}$ decreasing and $\mathrm{PaCO}_{2}$ possibly increasing). Rather than concentrating on values of $\mathrm{PetCO}_{2}$, which do not necessarily reflect $\mathrm{PaCO}_{2}$, we sought to interpret the capnogram shape.

There are certain weaknesses in this study that we must acknowledge. First, there were a few patients with a large number of medical conditions; second, the normal morphology (non-wheezing) and the obstructed morphology (wheezing) group were not closely matched for sex and age; however, there is no evidence to suggest that capnogram shape is dependent on age or $\operatorname{sex}^{1-3}$; finally, we were disappointed that many of the expiratory capnograms, particularly in the wheezing group, were unsuitable for slope ratio analysis, usually because of an inadequate cycle length (due to tachypnoea) or because of deformation by artefact. In addition, airway obstruction from other causes, such as nasal oedema and nasal obstruction, may result in an obstructive pattern on capnogram traces. It may be expedient in such cases to get the patient to breathe for a number of cycles through the mouth, through the open port of a conventional capnogram endotracheal tube fitting.

\section{CONCLUSIONS}

Nasal capnography can be used as a means of respiratory monitoring in non-intubated patients in an emergency medicine setting. It is non-invasive, does not rely heavily on patient compliance, does not necessitate the interruption of oxygen or nebulised drug treatment, and provides continuous real time information in a simple graphic form. Further studies with larger matched patient populations and therapeutic outcome analysis would be helpful in further determining the clinical usefulness of capnography in this setting.

1 O'Flaherty. Capnography: principles and practice. London BMJ Publishing Group, 1994

2 Smalhout B, Kalenda Z. An atlas of capnography.

Amsterdam: Kerchebosch-Zeist, 1981. Weingarten M. Respiratory monito

tive. J Clin Monit 1990;6:217-25.
You B, Peslin R, Duvivier C, Dang Vu V, Grilliat JP. ExpiraYou B, Peslin R, Duvivier C, Dang Vu V, Grilliat JP. Expira-
tory capnography in asthma: evaluation of various shape tory capnography in asthma: evaluati
indices. Eur Resp J 1994;7:318-23.

5 Barach EM. Asthma in ambulatory care: use of objective diagnostic criteria. J Fam Pract 1994;38:161-5.

6 You B, Mayeux D, Rkiek B, Autran N, Dang Vu V, Grillat JP. La capnographie expiratoire dans l'asthme: perspectives d'utilisation comme méthode de monitorage. Rev Mal Respir 1992;9:547-52.

7 Plewa MC, Sikora S, Engoren M, Tome D, Thomas J, Deuster A. Evaluation of capnography in non-intubated emergency department patients. Acad Emerg Med 1995;2: 901-8. 